INPLASY

PROTOCOL

To cite: Sacco. Genetic and pharmacogenomic aspects of the medication related osteonecrosis of the jaw (MRONJ): a quality research umbrella review. Inplasy protocol 202230002. doi: 10.37766/inplasy2022.3.0002

Received: 01 March 2022

Published: 01 March 2022

Corresponding author:

Roberto Sacco

roberto.sacco@manchester.ac.uk

Author Affiliation:

Roberto Sacco

Support: No Financial Support.

Review Stage at time of this submission: Data extraction.

Conflicts of interest:

None declared.

\section{Genetic and pharmacogenomic aspects of the medication related osteonecrosis of the jaw (MRONJ): a quality research umbrella review}

Sacco, R1.

Review question / Objective: What is the current state of evidence related to genetic and pharmacogenomic associated to MRONJ? - Population (P): any (no limits of age) patients with MRONJ - Interventions (I): any types Comparison (C): any types - Outcome (O): Quality evaluation of the high ranked articles in literature (systematic review and meta-analysis) in relationship with the genetic and pharmacogenomics aspects associated with MRONJ - Study (S): systematic review (SR) or meta-analysis (MA).

Condition being studied: Medication related osteonecrosis of the jaw (MRONJ) is an irreversible adverse event related principally to antiresorptive medications (e.g. bisphosphonates and receptor activator of nuclear factor Kappa-B ligand inhibitors) and angiogenesis inhibitors. These types of drug therapies are used for the treatment of the skeletal manifestation of malignancies and/or bone metastases and in the management of osteoporosis, Paget's disease or hypercalcemia. The pathogenesis of MRONJ remains unclear, however, a number of risk factors have been identified that are associated with MRON J development. Genetic predisposition for MRONJ has been investigated in recent years with confounding results. Hence the need of evaluating the quality level of research associated with it.

INPLASY registration number: This protocol was registered with the International Platform of Registered Systematic Review and Meta-Analysis Protocols (INPLASY) on 01 March 2022 and was last updated on 01 March 2022 (registration number INPLASY202230002).

\section{INTRODUCTION}

Review question / Objective: What is the current state of evidence related to genetic and pharmacogenomic associated to
MRONJ? - Population (P): any (no limits of age) patients with MRONJ - Interventions (I): any types - Comparison (C): any types Outcome (0): Quality evaluation of the high ranked articles in literature (systematic 
review and meta-analysis) in relationship with the genetic and pharmacogenomics aspects associated with MRONJ - Study (S): systematic review (SR) or metaanalysis (MA).

Condition being studied: Medication related osteonecrosis of the jaw (MRONJ) is an irreversible adverse event related principally to antiresorptive medications (e.g. bisphosphonates and receptor activator of nuclear factor Kappa-B ligand inhibitors) and angiogenesis inhibitors. These types of drug therapies are used for the treatment of the skeletal manifestation of malignancies and/or bone metastases and in the management of osteoporosis, Paget's disease or hypercalcemia. The pathogenesis of MRONJ remains unclear, however, a number of risk factors have been identified that are associated with MRON J development. Genetic predisposition for MRONJ has been investigated in recent years with confounding results. Hence the need of evaluating the quality level of research associated with it.

\section{METHODS}

Search strategy: A search strategy for all databases was developed as follows:

1. Osteonecrosis [MeSH Terms] OR Avascular osteonecrosis of the jaw [MeSH Terms] OR Osteonecrosis of the jaw [MeSH Terms] OR MRONJ [MeSH Terms] OR ONJ [MeSH Terms] OR BONJ [MeSH Terms] OR ARONJ [MeSH Terms] OR BRONJ [MeSH Terms] OR Pharmacogenetics [MeSH Terms] OR Genetic predisposition [MeSH Terms] OR Pharmacogenomics

2. Patients [MeSH Terms] OR Any patients [MeSH Terms] OR Oncology [MeSH Terms] OR Osteoporosis [MeSH Terms] OR Nononcologic patients

3. Systematic review [MeSH Terms] OR Review [MeSH Terms] OR Meta-analysis

4. 1 and 2 and 3.

Participant or population: Any (no limits of age) patients with MRONJ.

Intervention: Any types.
Comparator: Any types.

Study designs to be included: Systematic review (SR) or meta-analysis (MA).

Eligibility criteria: The search strategy for this study will be focused on published systematic reviews and/or meta-analysis. No language restrictions will be imposedon the search. Narrative reviews, animal reviews, and those reviews which included patients with a previous history of radiation therapy to the head and/or neck regions will be excluded.

Information sources: PubMed, MEDLINE, EMBASE and CINAHL.

Main outcome(s): Evaluate the current quality of the state of knowledge regarding the medication-related osteonecrosis of the jaw as it relates to the potential genetic and pharmacogenomics characteristics in SR and MA.

Additional outcome(s): Evaluate other factors such as: - Type of studies included in the systematic reviews (SR) and/or metaanalysis (MA) - Number of patients included in the reviews - Type of patients evaluated in the studies (Oncology or Non-oncology).

Quality assessment / Risk of bias analysis: All selected papers will be carefully read by independent review authors and data will be extracted using a research report form. The number of included studies, design of the studies, number of patients, results, quality of the evidence, and recommendation will be recorded. In case of missing information, authors will be contacted and given 6 weeks to respond.

Strategy of data synthesis: Following the screening of the eligible studies, all selected papers will be carefully read to identify the study and to assess primary and secondary outcomes. Data will be extracted from each study and analyzed. This will be done independently by selected authors. Where pooling of results is inappropriate, the results will be reported as narrative descriptions using a detailed commentary. 
Subgroup analysis: All participants will be included in the final analysis. If data permits, a subgroup analysis will be included in this review.

Sensitivity analysis: If sufficient data are extracted, a sensitivity analysis will be conducted to check the stability of the outcome results by excluding low the methodological quality or high risk of bias studies.

Language: No language restrictions will be applied.

Country(ies) involved: United Kingdom.

Keywords: osteonecrosis; medication related osteonecrosis of the jaw, genetic, pharmacogenomics, umbrella review, evidence base medicine.

Contributions of each author:

Author 1 - Roberto Sacco.

Email: roberto.sacco@manchester.ac.uk 